\title{
Expression of cyclooxygenase-2 (COX-2) in tumour and stroma compartments in cervical cancer: clinical implications
}

\section{G Ferrandina', L Lauriola ${ }^{2}$, GF Zannoni' ${ }^{2}$ MG Distefano', F Legge', V Salutari', M Gessi' ${ }^{2}$ N Maggiano², G Scambia' and FO Ranelletti*,3}

'Department of Obstetrics and Gynecology, Catholic University of the Sacred Heart, L.go F. Vito, I, 00168 Rome, Italy; ${ }^{2}$ Department of Pathology, Catholic University of the Sacred Heart, L.go F. Vito, I, 00168 Rome, Italy; ${ }^{3}$ Department of Histology, Catholic University of the Sacred Heart, L.goF. Vito, I, 00168 Rome, Italy

This study aims at investigating the relationship between cyclooxygenase-2 expression in tumour vs stroma inflammatory compartment and its possible clinical role. The study included 99 stage IB-IV cervical cancer patients: immunostaining of tumour tissue sections was performed with rabbit antiserum against cyclooxygenase-2. CD3, CD4, CD8, CD25, Mast Cell Tryptase monoclonal antibodies were used to characterise stroma inflammatory cells in nine cervical tumours. An inverse relation was found between cyclooxygenase-2 levels (cyclooxygenase-2 IDV) of tumour vs stroma compartment $(r=-0.44$, $P<0.000$ I). The percentage of cases showing high tumour/stromal cyclooxygenase-2 IDV ratio was significantly higher in patients who did not respond to treatment (93.3\%) with respect to patients with partial (60.5\%), and complete (43.7\%) response $(P=0.009)$. Cases with a high tumour/stroma cyclooxygenase-2 IDV ratio had a shorter overall survival rate than cases with a low tumour/stroma cyclooxygenase-2 IDV $(P<0.000 \mathrm{I})$. In the multivariate analysis advanced stage and the status of tumour/stroma cyclooxygenase-2 IDV ratio retained an independent negative prognostic role. The proportion of $\mathrm{CD}^{+}$, $\mathrm{CD}^{+}$, and $\mathrm{CD} 25^{+}$cells was significantly lower in tumours with high tumour/stroma cyclooxygenase-2 IDV ratio, while a higher percentage of mast cells was detected in tumours showing high tumour/stroma cyclooxygenase-2 IDV ratio. Our study showed the usefulness of assessing cyclooxygenase- 2 status both in tumour and stroma compartment in order to identify cervical cancer patients endowed with a very poor chance of response to neoadjuvant therapy and unfavourable prognosis. British Journal of Cancer (2002) 87, I | 45 - I I52. doi: I0. I038/sj.bjc.6600578 www.bjcancer.com

(c) 2002 Cancer Research UK

Keywords: cervical cancer; COX-2; prognosis; chemotherapy response

Cyclooxygenase (COX), the key enzyme in the conversion of arachidonic acid to prostaglandins, exists in two isoforms namely COX-1, constitutively expressed in most tissues, and COX-2, which is inducible by growth factors, prostaglandins, and tumour promoters and associated with the inflammatory response (Williams and Dubois, 1996). It has been reported that COX-2 overexpression is associated with inhibition of apoptosis and host immune responses, and increased metastatic potential and neoangiogenesis (Tsujii and Dubois, 1995; Tsujii et al, 1998; Stolina et al, 2000). In this context, it is not surprising that COX-2 has been associated with parameters of tumour aggressiveness and unfavourable clinical outcome in several solid tumours (Ferrandina et al, 2002a; Tomozawa et al, 2000; Ristimaki et al, 2002). As far as cervical cancer is concerned, recent reports showed that high COX-2 expression is associated with diminished survival in cervical cancer patients administered radiotherapy (Gaffney et al, 2001). We also recently demonstrated that high COX-2 expression in tumour cells can identify cervical cancer patients with a poor chance of response to neoadjuvant chemotherapy and worse prognosis (Ferrandina et al, 2002b).

There is some evidence that, besides the expression in tumour cells, COX-2 is also expressed in stromal cellular elements such

*Correspondence: Professor FO Ranelletti; E-mail: ranelletti@rm.unicatt.it Received 3 April 2002; revised 25 July 2002; accepted 15 August 2002 as endothelial cells, macrophages, and fibroblasts (Masferrer et al, 2000), stimulated T-lymphocytes and activated mast cells (Iniguez et al, 1999; Hundley et al, 2001).

The potential role of COX-2 expression in stroma cells has been first suggested by Williams et al (2000), who showed a dramatic inhibition of tumour growth and angiogenesis in lung carcinoma cells grafted into homozygous COX-2 $2^{--}$mice.

Based on our preliminary observations about the presence of COX-2 staining in the stroma compartment of cervical tumours we were then prompted at providing a more in depth analysis of (i) COX-2 content in the stroma inflammatory cellular elements of this neoplasia, (ii) the relationship between COX-2 expression in tumour cells $v s$ stroma inflammatory compartment, and (iii) the possible clinical role of COX-2 expression according to the cellular compartment of staining.

In addition, an immunophenotypic characterization of stroma inflammatory cells was carried out in a series of cervical tumours.

\section{PATIENTS AND METHODS}

The study included 99 stage IB-IV cervical cancer patients consecutively admitted to the Department of Obstetrics and Gynecology, Division of Gynecologic Oncology, Catholic University of Rome between November 1995 and September 2001. Median age was 51 years (range 24-76). The clinico-pathological characteristics are summarised in Table 1 . The clinical management of our patient 
Table I Distribution of tumour/storma COX-2 IDV ratio according to clinico-pathological characteristics of cervical cancer population

\begin{tabular}{|c|c|c|c|c|c|}
\hline Characteristics & $\begin{array}{l}\text { No. of } \\
\text { patients }\end{array}$ & $\begin{array}{c}\text { High tumour/stroma } \\
\text { COX-2 IDV ratio } \\
\text { no. (\%) }\end{array}$ & $P$ value ${ }^{a}$ & $\begin{array}{l}\text { Tumour/stroma } \\
\text { COX-2 IDV ratio } \\
\text { (mean } \pm \text { s.e.) }\end{array}$ & $P$ value $^{\mathrm{b}}$ \\
\hline All cases & 99 & $56(56.6)$ & & $5.1 \pm 0.9$ & \\
\hline $\begin{array}{l}\text { Age (years) } \\
\quad<50 \\
\quad \geqslant 50\end{array}$ & $\begin{array}{l}46 \\
53\end{array}$ & $\begin{array}{l}23(50.0) \\
33(62.3)\end{array}$ & 0.30 & $\begin{array}{l}4.3 \pm 1.1 \\
5.7 \pm 1.3\end{array}$ & 0.12 \\
\hline $\begin{array}{l}\text { FIGO stage } \\
\text { IB } \\
\| \\
\text { III-IV }\end{array}$ & $\begin{array}{l}25 \\
56 \\
18\end{array}$ & $\begin{array}{l}\text { II (44.0) } \\
30(55.6) \\
\text { I5 (83.3) }\end{array}$ & 0.029 & $\begin{array}{l}4.0 \pm 1.3 \\
4.9 \pm 1.2 \\
7.0 \pm 2.3\end{array}$ & 0.09 \\
\hline $\begin{array}{l}\text { Histotype } \\
\text { Squamous } \\
\text { Adenocarcinoma } \\
\text { Adenosquamous }\end{array}$ & $\begin{array}{c}81 \\
12 \\
6\end{array}$ & $\begin{array}{c}41(50.6) \\
12(100) \\
3(50)\end{array}$ & $0.052^{c}$ & $\begin{array}{l}3.9 \pm 0.8 \\
12.9 \pm 4.4 \\
5.6 \pm 2.5\end{array}$ & $0.0005^{c}$ \\
\hline $\begin{array}{l}\text { Grade } \\
\qquad \begin{array}{l}1-2 \\
3 \\
\text { Not available }\end{array}\end{array}$ & $\begin{array}{l}37 \\
52 \\
10\end{array}$ & $\begin{array}{l}23(62.0) \\
27(51.9)\end{array}$ & 0.84 & $\begin{array}{l}4.7 \pm 1.3 \\
4.6 \pm 1.2 \\
-\end{array}$ & 0.89 \\
\hline $\begin{array}{l}\text { Tumour size }(\mathrm{cm}) \\
\quad<4 \\
\geqslant 4\end{array}$ & $\begin{array}{l}37 \\
62\end{array}$ & $\begin{array}{l}\text { I5 (40.5) } \\
4 \mid(66.1)\end{array}$ & 0.023 & $\begin{array}{l}2.4 \pm 0.6 \\
6.6 \pm 1.3\end{array}$ & 0.011 \\
\hline $\begin{array}{l}\text { Clinical response } \\
\text { Complete } \\
\text { Partial } \\
\text { NC/P }\end{array}$ & $\begin{array}{l}16 \\
43 \\
16\end{array}$ & $\begin{array}{l}7(43.7) \\
26(60.5) \\
15(93.7)\end{array}$ & 0.009 & $\begin{array}{l}4.2 \pm 2.1 \\
4.9 \pm 1.1 \\
10.6 \pm 3.6\end{array}$ & 0.032 \\
\hline
\end{tabular}

population was as previously described (Ferrandina et al, 2002b): cases with early stage disease (FIGO Stage IB-IIA, major tumour diameter less than $4 \mathrm{~cm}$ ) were primarily submitted to radical surgery $(n=24)$, whereas locally advanced cancer cases $(n=75)$ were first administered neoadjuvant cisplatin-based treatment (cisplatin dose $100 \mathrm{mg} \mathrm{m}^{2}$ every 3 weeks for $2-3$ courses). In case of clinical response, assessed by the above described procedures and recorded according to WHO response evaluation criteria (World Health Organization, 1979), LACC patients were submitted to radical surgery $(n=61)$ : operative technique consisted of type II - IV radical hysterectomy, and systematic pelvic lymphadenectomy. An anterior exenteration was performed in two cases with persisting involvement of the bladder after neoadjuvant treatment. Para-aortic lymphadenectomy up to the level of inferior mesenteric artery was performed in high risk patients as previously reported (Benedetti-Panici et al, 1996). Patients showing clinical no change/ progression during neoadjuvant treatment were subjected to exclusive radiotherapy.

\section{Antibodies}

The following monoclonal and policlonal antibodies were used: anti-CD3 (clone PS1; 1:100), anti-CD4 (clone 1F6; 1:50), antiCD8 (clone 4B11; 1:50), anti-CD25 (clone 4C9; 1:100), all from Ylem (Avezzano, Italy); anti-Mast Cell Tryptase (clone AA1, 1:100 (DAKO, Glostrup, Denmark); anti-COX 2 rabbit policlonal antibody (1:300 Cayman, Ann Arbor, MI, USA).

\section{Immunohistochemical studies}

Tumour tissues biopsies were performed under colposcopic examination. Immunohistochemistry of COX-2 was performed as previously described (Ferrandina et al, 2002b). In the studies of phenotyping of stromal inflammatory cells, serial sections of repre- sentative blocks were cut from patients with either high $(>1)$ or low $(\leqslant 1)$ COX-2 positive tumour/stroma ratio (see below). In order to quantitate the percentage of COX-2 stained cell subpopulations with respect to inflammatory cells of the stroma, we used the immunoperoxidase technique performed on consecutive sections, since it allows by nuclear counterstaining with hematoxylin to better recognise stromal cells also by their morphological characteristics.

Negative controls were performed using non immunised rabbit serum and/or omitting the primary antibodies. As positive controls for COX-2 antibody, COX-2 positive Hep-2 squamous cancer cells and COX-2 positive squamous cancer tissue specimens (Ranelletti et al, 2001) were always run in the assay. As positive controls for the other antibodies both lymph-nodes and thymus were used.

\section{Double-labelling subtraction immunostaining}

Double-labelling subtraction immunostaining was carried out as previously reported (Yamada et al, 1998). Briefly, after routine immunostaining for COX-2, the slide was treated with 3-amino-9 ethylcarbazole (Sigma, St. Louis, MO, USA). The slide was rapidly mounted using an aqueous mounting medium and digital images of microscopic fields acquired by Nikon Coolpix 950 digital camera (Nikon Instruments, Florence, Italy) after registering the X-Y coordinate of the acquired field in the slide. Then the slide was

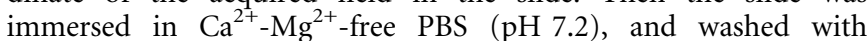
PBS. The slide was immersed for $10 \mathrm{~s}$ in $99.8 \%$ (vol/vol) methyl alcohol and then exhaustively washed with PBS. The section was treated in microwave owen using the Dako ChemMate detection kit (DAKO) and then incubated with anti-mast cell tryptase antibody. The immunoreaction was developed by fast blue and the slide was remounted. The exact area on the same slide was acquired by the digital camera and compared with the first image. As a control, after the first immunostaining with anti-COX-2 anti- 

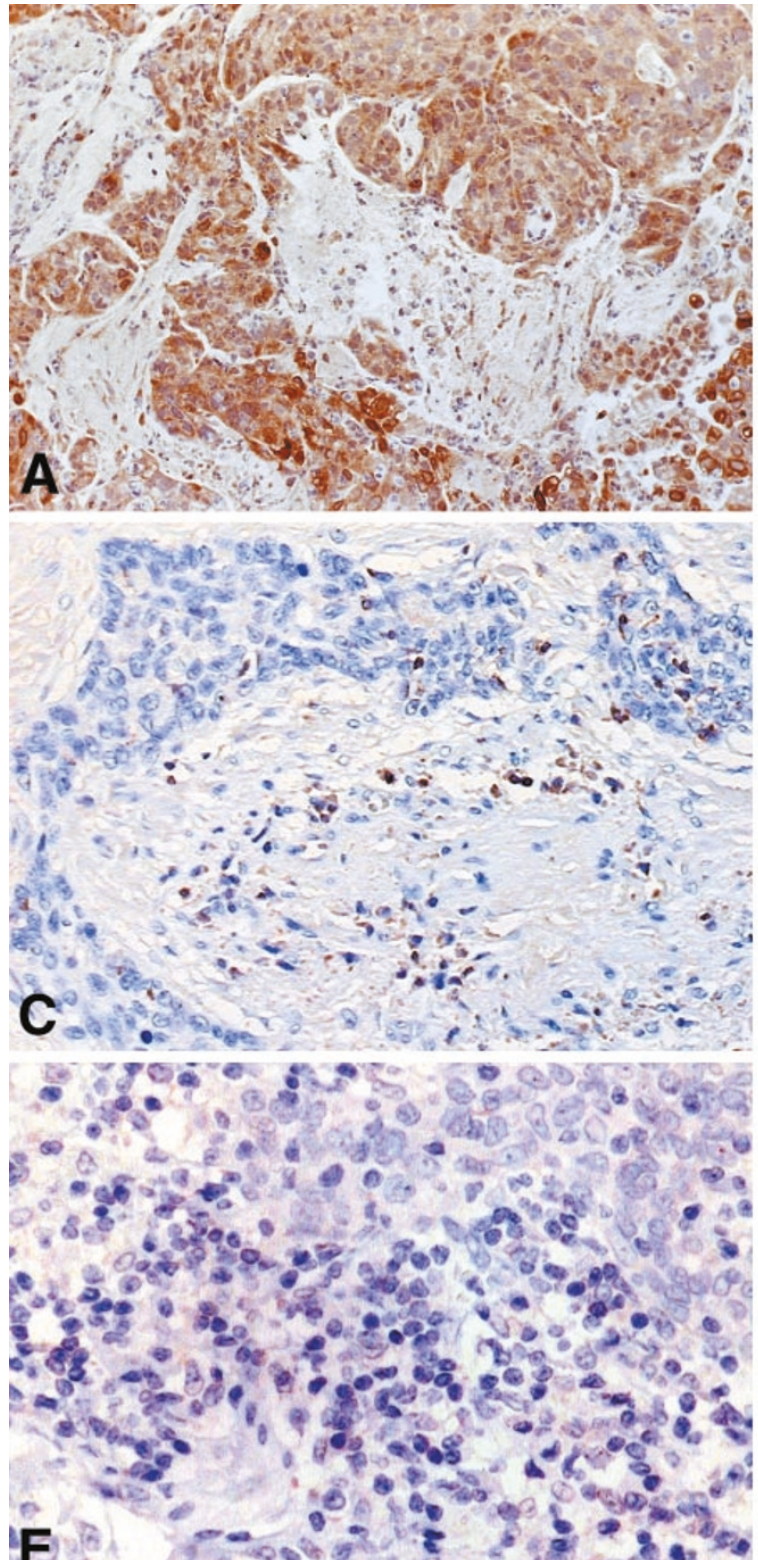

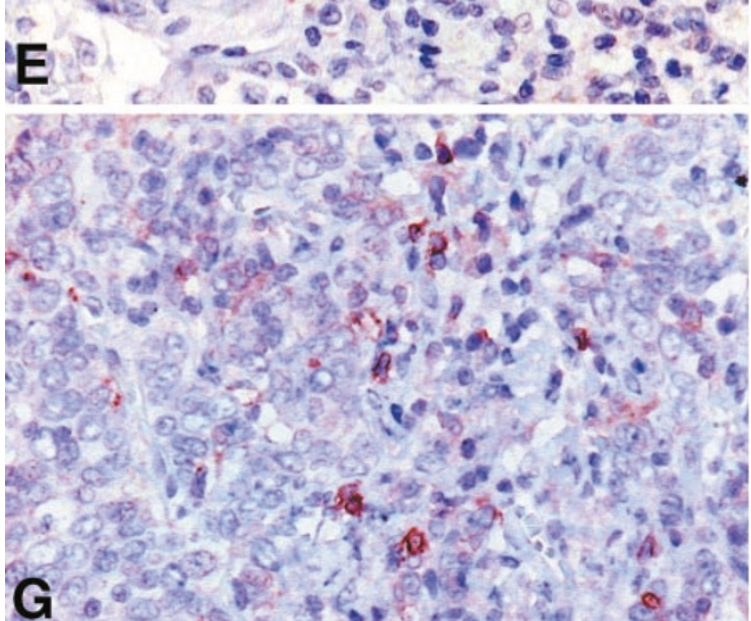

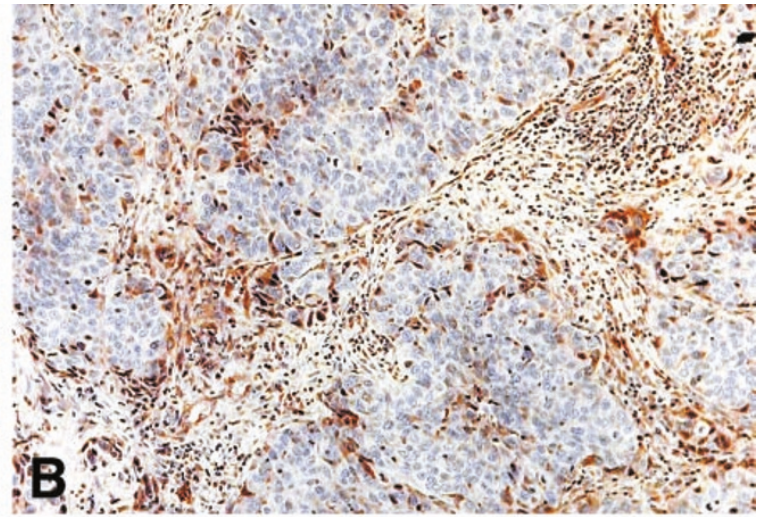

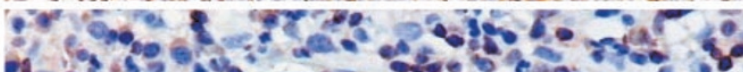

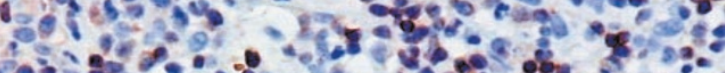

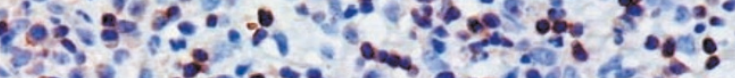

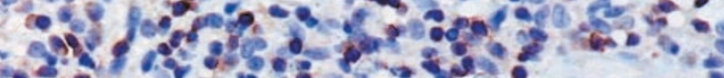
is dif

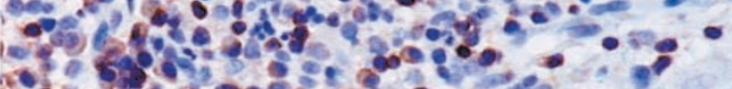

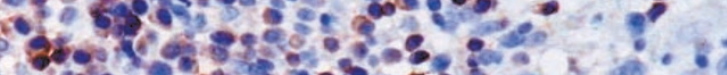

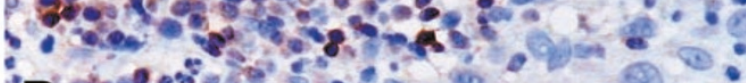

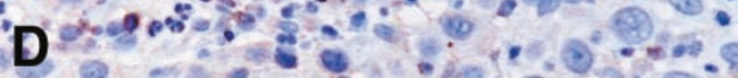

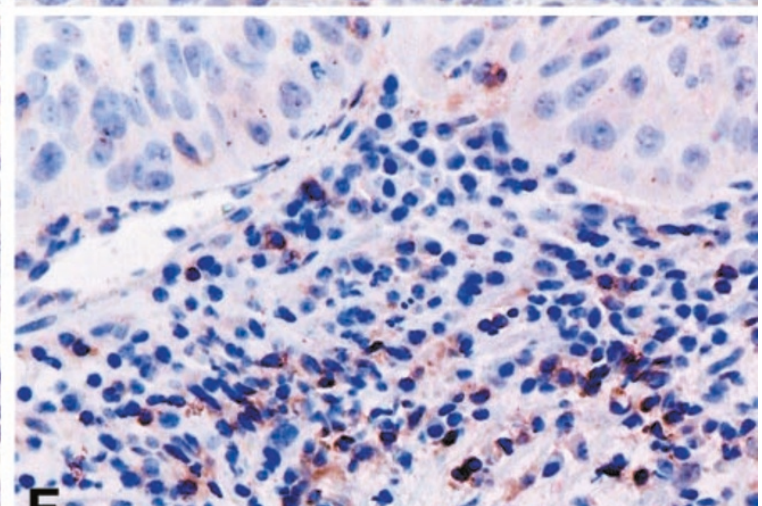
F-

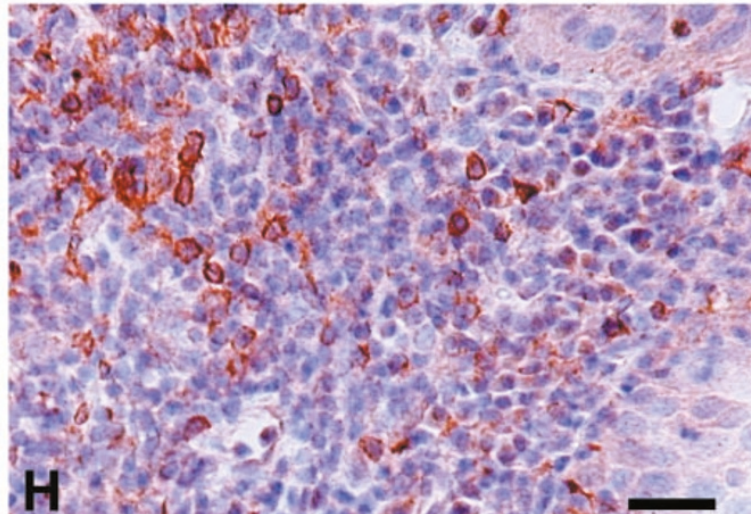

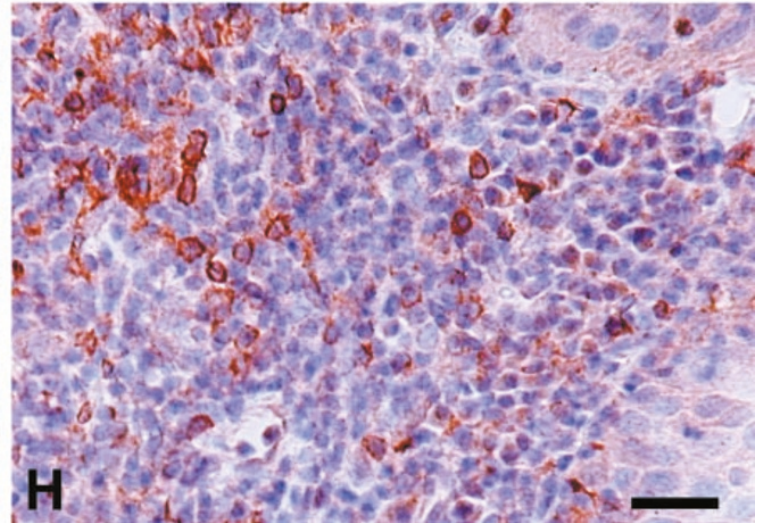

Figure I (A) Squamous cervical cancer with intense COX-2 immunoreaction in both cytoplasm and nuclei of tumour cells. Scattered cells in the stromal compartment are stained. (B) COX-2 negative tumour showing intense COX-2 staining in the stroma inflammatory compartment. CD3, CD4, CD25, and tryptase immunoreaction in tumours showing high $(\mathbf{C}, \mathbf{E}, \mathbf{G})$ vs low $(\mathbf{D}, \mathbf{F}, \mathbf{H})$ tumour/stroma COX-2 IDV ratio. Bar=50 $\mu \mathrm{m}(\mathbf{A}, \mathbf{B}) ; \operatorname{Bar}=25 \mu \mathrm{m}(\mathbf{C}-\mathbf{H})$. 
body, the slide was processed, as reported above, and then treated with the streptavidin-biotin-alkaline phosphatase complex kit, omitting the anti-mast cell tryptase antibody.

\section{Quantification of immunohistochemical staining}

The intensity of immunohistochemical staining was evaluated as previously reported (Ranelletti et al, 2001) by the image analysis based on Photoshop (Adobe System, San Jose, CA, USA) together with 'The image processing toolkit' (CRC Press, Boca Raton, FL, USA). The integrated density values (IDV) of the immunostaining was calculated as the product of the mean density value of the immunoreactive regions by the percentage of the immunostained tumour or stroma components. Inflammatory cell count in the tumour stroma was performed by chosing five corresponding $\times 20$ fields, from each of six serial tissue sections (one for each antibody), so as to best reflect the overall immunostaining of the tumour stroma contained in the entire slide. The files, acquired with a Nikon Coolpix 950 digital camera, were opened in Photoshop using a Macintosh G3 workstation (Apple, Cupertino, CA, USA). Both immunostained and negative cells within a superimposed grid of $0.022 \mathrm{~mm}^{2}$ were counted. Two cell counts for each digital image were done by moving the grid over representative stromal areas. The total cell number/stromal area was calculated by averaging cell counts from each section and from the six consecutive sections $(n=60)$. The number of immunostained cells, relative to each phenotype, was calculated by averaging cell counts from two grid areas from five $\times 20$ fields $(n=10)$. The results were reported as mean \pm s.e.

\section{Statistical analysis}

The $\chi^{2}$-test was used to analyse the distribution of COX-2 positive cases according to several clinico-pathological features. KruskalWallis non parametric test was used to analyse the distribution of tumour/stroma COX-2 integrated density values according to clinico-pathological variables. Medians and life tables were computed using the product-limit estimate by the Kaplan and Meier method (Kaplan and Meyer, 1958) and the log-rank test was employed to assess the statistical significance (Mantel, 1966). The prognostic role of COX-2 as a continuous variable, was also analysed by means of the Cox proportional hazard model (Cox, 1972). A Cox's regression model with stepwise variable selection (Cox, 1972) and multiple logistic analysis (Cox, 1970) were used to analyse the role of clinico-pathological parameters and COX-2 staining as prognostic factors and predictors of response to neoadjuvant treatment. Wald statistics for coefficient comparison and the Joint significance test were also performed in order to compare the coefficients of relative risk of death for tumour COX-2 positivity $v s$ tumour/stroma COX-2 IDV ratio positivity and to evaluate the weight of the status of tumour COX-2 and tumour/stroma COX-2 IDV ratio in the survival regression model after excluding each of them.

Statistical analysis was carried out using SOLO (BMDP Statistical Software, Los Angeles, CA, USA) and Statview survival tools (Abacus Concepts- Inc- Berkeley CA, USA).

\section{RESULTS}

\section{Cox-2 immunostaining}

Figure $1 \mathrm{~A}$ and $\mathrm{B}$ shows COX-2 immunoreaction in two primary squamous cervical tumours. COX-2 immunostaining was observed both in the tumour cells as well as in the stroma inflammatory compartment of the tumour. Interestingly, in the presence of strong COX-2 staining in tumour cells, only barely detectable COX-2 immunoreaction was observed in the stroma inflammatory compartment (Figure 1A). On the other hand, a large amount of stroma inflammatory component showing positive COX-2 immunostaining was frequently detected in association with low or absent COX-2 staining in tumour cells (Figure 1B).

In the whole series, COX-2 integrated density values in the tumour component ranged from 1.2 to 82.3 with mean \pm s.e. values $25.5 \pm 2.2$. COX-2 integrated density values in the stromal component range from 0.9 to 96.0 with mean+s.e. values of $20.0 \pm 1.9$.

A statistically significant inverse relation was found between COX-2 IDV of tumour $v s$ COX-2 IDV in the stroma compartment $(r=-0.44, P<0.0001)$ (data not shown).

For this reason, the ratio between COX-2 IDV in the tumour $v$ s COX-2 IDV in the stroma component was used in order to normalise the COX-2 expression in each case, and to categorise tumours according to low vs high COX-2 content. The tumour/ stroma COX-2 IDV ratio range from 0.03 to 48.2 (mean \pm s.e. $=5.1 \pm 0.9$ ). The ratio of $\leqslant 1$ was used to indicate cervical tumours with COX-2 expression in the tumour component lower or equivalent to COX-2 expression in the stroma.

According to the chosen cut off value, 56 out of $99(56.6 \%)$ were scored as having a high $(>1)$ tumour/stroma COX-2 IDV ratio.

\section{Correlation with clinico-pathological parameters}

High COX-2 IDV in the tumour compartment were shown to be significantly associated with larger volume of the tumour and more aggressive histotype while COX-2 IDV in the tumour stroma showed the opposite pattern (data not shown).

The percentage of cases with high tumour/stroma COX-2 IDV ratio increased from $44.0 \%$ in stage I, through $55.6 \%$ in stage II, to $83.3 \%$ in stage III - IV cases ( $P$ value $=0.029)$. Moreover, cases with high tumour/stroma COX-2 IDV ratio were more frequently observed in cases with tumour volume $\geqslant 4 \mathrm{~cm}$ than in smaller tumours $(66.1 \%$ vs $40.5 \%)$ ( $P$ value $=0.023)$. No association with age, and grade of differentiation was found (Table 1).

Similarly, higher tumour/stroma COX-2 IDV ratio was found in stage III-IV with respect to stage I-II cases $(P$ value $=0.09)$, in adenocarcinoma and adenosquamous carcinoma versus squamous cell $(P$ value $=0.0005)$, in tumours $\geqslant 4 \mathrm{~cm}$ vs smaller tumours $(P$ value $=0.011)$.

Metastatic lymph node involvement was found in 14 out of 69 $(20.3 \%)$ cases: the percentage of COX-2 tumour positivity was $28.6 \%$ in lymph node positive with respect to $35.7 \%$ in lymph node negative cases (difference not significant).

\section{COX-2 status and response to neoadjuvant treatment}

The percentage of cases showing tumour COX-2 positivity was significantly higher in patients who did not respond to treatment $(87.5 \%)$ with respect to patients with partial $(46.5 \%)$, and complete $(31.2 \%)$ response $(P=0.003)$. Similar results were found considering COX-2 IDV ratio as covariate, according to clinical response $(P=0.007)$ (data not shown). On the other hand, the percentage of cases showing COX-2 IDV positivity in the stroma was not correlated per se with response to treatment (data not shown).

The percentage of cases showing high tumour/stromal COX-2 IDV ratio was significantly higher in patients who did not respond to treatment $(93.3 \%)$ with respect to patients with partial $(60.5 \%)$, and complete $(43.7 \%)$ response $(P=0.009)$. Similar results were found considering mean COX-2 IDV ratio according to clinical response (Table 1).

In the univariate analysis advanced FIGO stage, and high tumour/stroma COX-2 IDV ratio proved to associated with poor chance of response to neoadjuvant therapy (complete/partial vs no response). When logistic regression was applied, FIGO stage 
A

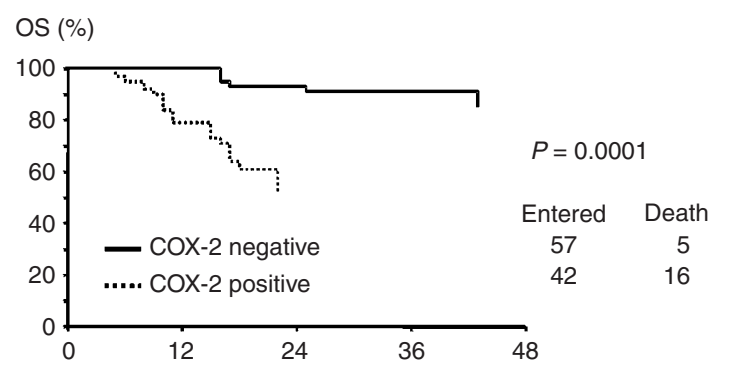

B

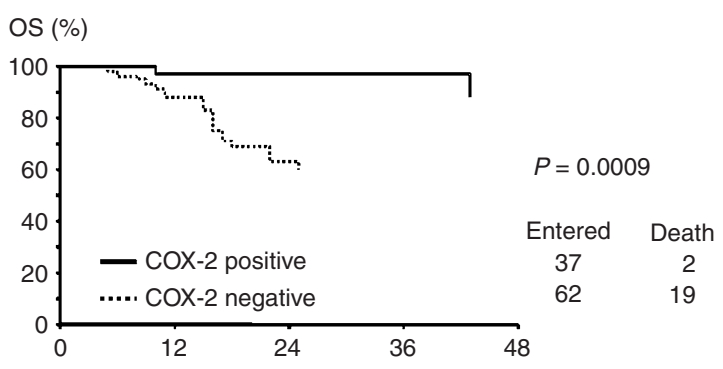

C

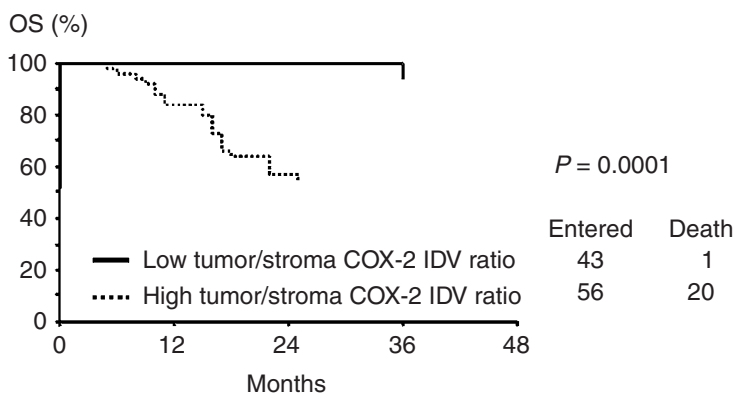

Figure 2 Overall survival rate according to the status of COX-2 in tumour cells $(\mathbf{A})$, in stroma inflammatory cells $(\mathbf{B})$, and according to the ratio between tumour/stroma COX-2 IDV $(\mathbf{C})$ in 99 cervical cancer patients.

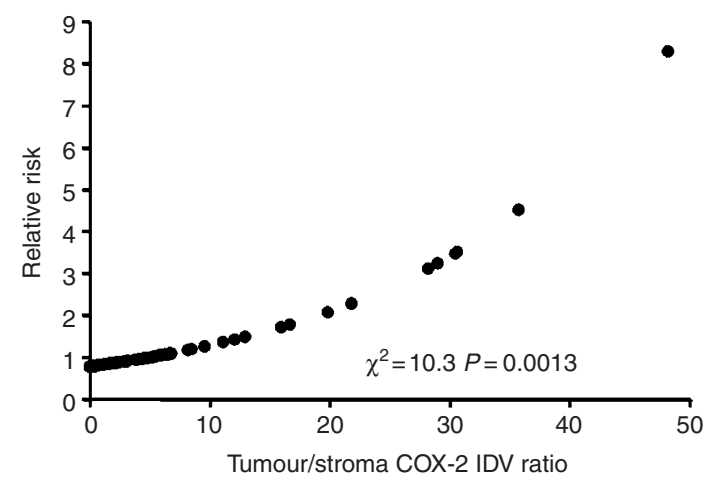

Figure 3 Plot of the estimate of the relative risk of death as a prediction of COX-2 integrated density values, calculated by COX's hazard regression model in cervical cancer patients.

$\left(\chi^{2}=7.0, P=0.008\right)$ and tumour/stroma COX-2 IDV ratio $\left(\chi^{2}=4.1\right.$, $P=0.0042)$ retained an independent role in predicting a poor chance of response to treatment.

\section{Survival analysis}

Follow up data were available for 99 patients. As of December 2001, the median follow up was 23 months (range=3-73). During the follow up period, 21 out of $99(21.2 \%)$ patients died of disease. For analysis of survival COX-2 status in tumour cells was defined according to the cut-off used in our previous study (Ferrandina et al, 2002b) and corresponding to the mean of COX-IDV. Similarly, COX-2 status in stroma inflammatory cells was defined according to the cut-off of 20.0 corresponding to mean COX-2 IDV.

Cases showing COX-2 positive reaction in tumour cells (Figure 2A) showed a shorter OS than COX-2 negative cases: in particular, the 3-year OS was 53\% (confidence intervals 95\% - CI 95\% 35-71) with respect to COX-2 negative cases who had a 3-year OS of $87 \%$ (CI $95 \%=75-99)(P=0.0001)$. COX-2 IDV in tumour cells were directly associated with risk of death, using COX-2 values as a continuous covariate $\left(\chi^{2}=16.6, P=0.0001\right.$ ) (data not shown).

On the other side, cases showing COX-2 positive reaction in stroma inflammatory cells showed a longer OS than COX-2 negative cases: in particular, the 3 -year OS was $91 \%$ (CI 95\%=79-101) in COX-2 positive with respect to COX-2 negative cases who had a 3 -year OS of 59\% (CI 95\%=46-72) $(P=0.010)$ (Figure 2B). COX-2 IDV in stroma inflammatory cells were inversely associated with risk of death, as assessed by COX analysis using COX-2 values as a continuous covariate $\left(\chi^{2}=8.2, P=0.004\right)$ (data not shown).

Cases with a high tumour/stroma COX-2 IDV ratio had a shorter OS than cases with a low tumour/stroma COX-2 IDV ratio: in particular, all deaths of disease but one occurred in the former group $(P<0.0001)$ (Figure $2 \mathrm{C})$. Similar results were observed in the subgroup of LACC patients (data not shown). The use of an arbitrary cut-off to distinguish cases with high $v s$ low tumour/stroma COX-2 IDV ratio is unlikely to have introduced any bias, since a direct association between tumour/stroma COX-2 IDV ratio values and risk of death in the overall series was found $\left(\chi^{2}=10.3\right.$; $P=0.0013)$ using the ratio between tumour/stromal COX-2 IDV as continuous covariate (Figure 3 ).

In multivariate analysis the positivity of tumour $\mathrm{COX}-2$ retained an independent negative prognostic role for OS $(P=0.0004)$, while the positivity of stromal COX-2 showed a trend toward a favourable role $(P=0.056)$. Similar results were obtained when using tumour COX-2 and stromal COX-2 as continuous variables (data not shown).

As shown in Table 2 the status of tumour/stroma COX-2 IDV ratio together with advanced stage of disease, retained an independent negative prognostic role for OS (Table 2). Similar results were obtained in multivariate analysis considering the values of tumour/ stroma COX-2 IDV ratio as continuous variable in the whole series as well as in LACC patients (data not shown). Finally, in order to evaluate the weight of the status of tumour COX-2 and tumour/ stroma COX-2 IDV ratio in the survival regression model, we tested the significance of excluding either the co-variate tumour COX-2 positivity or tumour/stroma COX-2 IDV positivity from the model. Indeed, only the tumour/stroma COX-2 IDV gave a statistically significant contribution to the model (likelihood ratio: $\chi^{2}=7.6, P=0.0058$ )

Moreover, the comparison between the coefficients of relative risk of death for tumour COX-2 positivity $v s$ tumour/stroma COX-2 IDV positivity was also analysed in order to test whether a statistically significant difference exists beween the two coefficients. Indeed, in the Cox's proportional hazard model we found that the relative risk of death in patients having a tumour/stroma COX-2 IDV ratio $>1$ positivity showed a trend to be higher (relative risk $18.72,95 \% \mathrm{CI}=1.94-181.0)$ than that of patients with tumour COX-2 (relative risk 1.12, 95\% CI=0.33-84.0) (Wald statistics: $\chi^{2}=3.292$; d.f. $\left.=1, P=0.069\right)$. 
Table 2 Univariate and multivariate analysis of clinico-pathological parameters and tumour/stroma COX-2 IDV ratio as prognostic factors in cervical cancer patients

\begin{tabular}{|c|c|c|c|c|c|c|}
\hline \multirow[b]{2}{*}{ Variable } & \multicolumn{3}{|c|}{ Univariate } & \multicolumn{3}{|c|}{ Multivariate $^{a}$} \\
\hline & RRI & $\chi^{2}$ & $P$ value & RR2 & $\chi^{2}$ & $P$ value \\
\hline $\begin{array}{l}\text { Age (years) } \\
<50\end{array}$ & $\stackrel{0}{0}$ & & & & & \\
\hline$\geqslant 50$ & 1.4 & 0.6 & 0.43 & - & - & - \\
\hline $\begin{array}{l}\text { Stage } \\
\mid-\|\end{array}$ & I음 & & & I은 & & \\
\hline$I I I-I V$ & 6.6 & 15.8 & 0.0001 & 3.5 & 6.2 & 0.012 \\
\hline $\begin{array}{l}\text { Histotype } \\
\text { Squamous } \\
\text { Other }\end{array}$ & $\begin{array}{l}100 \\
32\end{array}$ & 6.1 & 0.013 & $1 \stackrel{0}{21}$ & 2.1 & 0.14 \\
\hline $\begin{array}{l}\text { Tumour size } \\
\qquad 4 \mathrm{~cm} \\
\geqslant 4 \mathrm{~cm}\end{array}$ & $\begin{array}{l}10 \\
13.8\end{array}$ & 6.6 & 0.010 & $\begin{array}{l}\perp 0 \\
5.5\end{array}$ & 2.6 & 0.1 \\
\hline $\begin{array}{l}\text { Grade } \\
\text { GI -2 } \\
\text { G3 }\end{array}$ & $\begin{array}{l}10 \\
0.5\end{array}$ & 2.1 & 0.14 & $\begin{array}{l}\perp \stackrel{0}{ } \\
0.7\end{array}$ & 0.4 & 0.5 \\
\hline $\begin{array}{l}\text { Tumour/stroma COX-2 IDV ratio } \\
\text { Low } \\
\text { High }\end{array}$ & $\begin{array}{c}10 \\
21.5\end{array}$ & 8.9 & 0.0020 & $\begin{array}{c}10 \\
11.4\end{array}$ & 5.3 & 0.020 \\
\hline
\end{tabular}

${ }^{a}$ Only variables with $P$ value $<0.30$ in the univariate analysis were included in the multivariate model. RR I=unadjusted relative risk. RR2=relative risk after adjusting

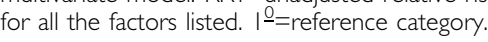

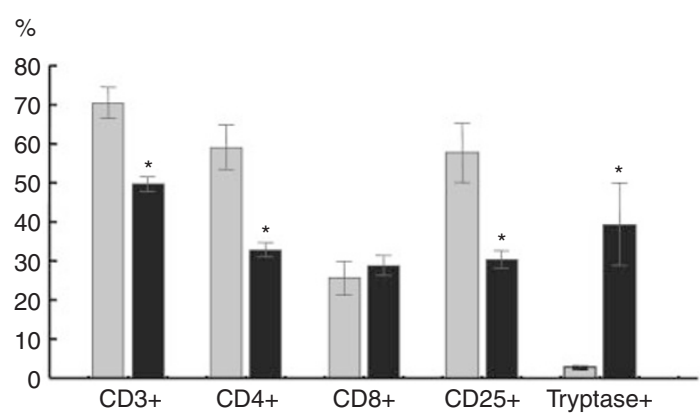

Figure 4 Percentage of $\mathrm{CD}^{+}, \mathrm{CD}^{+}, \mathrm{CD} 8^{+}, \mathrm{CD} 25^{+}$, and tryptase positive cells in stroma inflammatory infiltrate of cervical tumours from cases with low (gray columns) vs high (black columns) tumour/stroma COX-2 IDV ratio (results are expressed as mean \pm s.e.; * significant at $P<0.05$ ).

\section{Immunophenotypic characterisation}

Quantification of the total amount of cells as well as the expression of CD3, CD4, CD8, CD25 and tryptase in the stromal inflammatory compartment were analysed in nine squamous cervical cancers randomly chosen among cases with high $(>1)$ vs low $(\leqslant 1)$ tumour/stroma COX-2 IDV ratio. The total amount of cells observed in the stromal inflammatory compartment per unit area was significantly lower in cervical cancer showing high $v s$ low tumour/stroma COX-2 IDV ratio (mean \pm s.e. $=54.40 \pm 6.49$ vs $121.67 \pm 13.30, P=0.014)$. Moreover, analysis of the data of CD3, CD4, CD25, and tryptase immunostaining, represented in Figure $1 \mathrm{C}-\mathrm{H}$ and summarised in Figure 4, shows that the proportion of $\mathrm{CD}^{+}, \mathrm{CD}^{+}$, and $\mathrm{CD} 25^{+}$cells is significantly lower in tumours showing COX-2 positive vs COX-2 negative tumour cells $(P=0.014$; 0.025 , and 0.014 , for CD3, CD4, and CD25, respectively). On the contrary, a higher percentage of tryptase positive mast cells was detected in stromal inflammatory cells from tumours with COX2 positive $v s$ COX -2 negative tumour cells $(P=0.013)$. Interestingly, mast cells endowed with positive tryptase immunostaining were more frequently observed in tumours showing high versus low tumour/stroma COX-2 IDV ratio $(P=0.014)$.

Double labelling subtraction consecutive immunostaining of the same COX-2 positive tumour section by anti-COX-2 and anti-tryptase antibodies showed that the vast majority $(>70 \%)$ of tryptase positive mast cells also express COX-2 (Figure 5, insert).

\section{DISCUSSION}

In this study, we first reported that in cervical tumours the expression of COX-2 in the stroma inflammatory cells and its relationship with COX-2 expression in tumour cells can be clinically relevant. An inverse relationship between COX-2 expression in tumour cells and the amount of the stroma inflammatory infiltrate in the tumour has been detected.

Moreover, COX-2 positive tumours showed a lower percentage of COX-2 positive stromal cells/unit area. This finding seems to be a distinctive morphologic feature of cervical tumours since we could not find such histopathological pattern in ovarian (Ferrandina et al, 2002a) and endometrial cancer (manuscript submitted), and suggests that tissue specific factors may intervene to regulate tumour/host interactions and characterise the amount and possibly the composition of stromal cellular infiltrate. The association between the presence of an abundant lymphocytic infiltrate in the stroma and better clinical outcome has been already highlighted in cervical cancer (Chao et al, 1999) as well as other solid tumours (Zeid and Muller, 1993) and associated with the potentiation of antitumour immune response, although the subpopulations of immune cells involved have been only partially clarified (Chao et al, 1999; Santin et al, 2001). We showed a lower percentage of $\mathrm{CD}^{+}, \mathrm{CD}^{+}$and $\mathrm{CD} 25^{+} \mathrm{T}$ cells in tumours with COX-2 positive immunostaining in tumour cells suggesting that the expression of high levels of COX-2 in tumour cells can play a major role in inhibiting host immune functions. In this context, it is noteworthy that COX-2 can up-regulate the immunosuppressive cytokine IL10, while reducing the production of IL12, which is critical for cell mediated anti-tumour immunity (Stolina et al, 2000).

We also found a higher number of tryptase positive mast cells in tumours showing intense $v s$ low COX-2 staining in tumour cells. Moreover, a large proportion of stromal mast cells was found to express COX-2, suggesting that they are in an activated state, as supported by the observations that induction of COX-2 at mRNA and protein level occurs in antigen-activated mast cells (Hundley et al, 2001).

Tryptase positive mast cells have been reported to increase from normal cervical tissue through cervical dysplasias to invasive carcinoma (Bribiesca-Benitez et $a l, 2001$ ) and suggested to be involved in the promotion of tumour angiogenesis through the secretion of tryptase, a serin-protease able to exert a potent pro-angiogenic activity in human malignancies (Kankkunen et al, 1997).

Therefore, the presence of high COX-2 expression in cervical tumour cells seems to be associated with a scarce cellular infiltrate in the stroma and, notably, with a lower proportion of immunoregulatory cells and a high proportion of tryptase positive mast cells. At present, our data do not allow to indicate the mechanisms and direction of tumour/host interaction relative to COX-2 expression. The possible active role of COX-2 expression in the stromal compartment of the tumour in terms of tumour cell/ stromal cell cross-talk has been recently highlighted (Prescott, 2000). In particular, Lehay et al (2002) showed that induction of apoptosis by COX-2 inhibitors in angiogenic cells is associated with antitumour activity, suggesting that COX-2 expression in stromal elements such as the vasculature could play a major role in tumour biology. This issue is relevant in order to understand the role of COX-2 according to different compartments, and warrants further investi- 

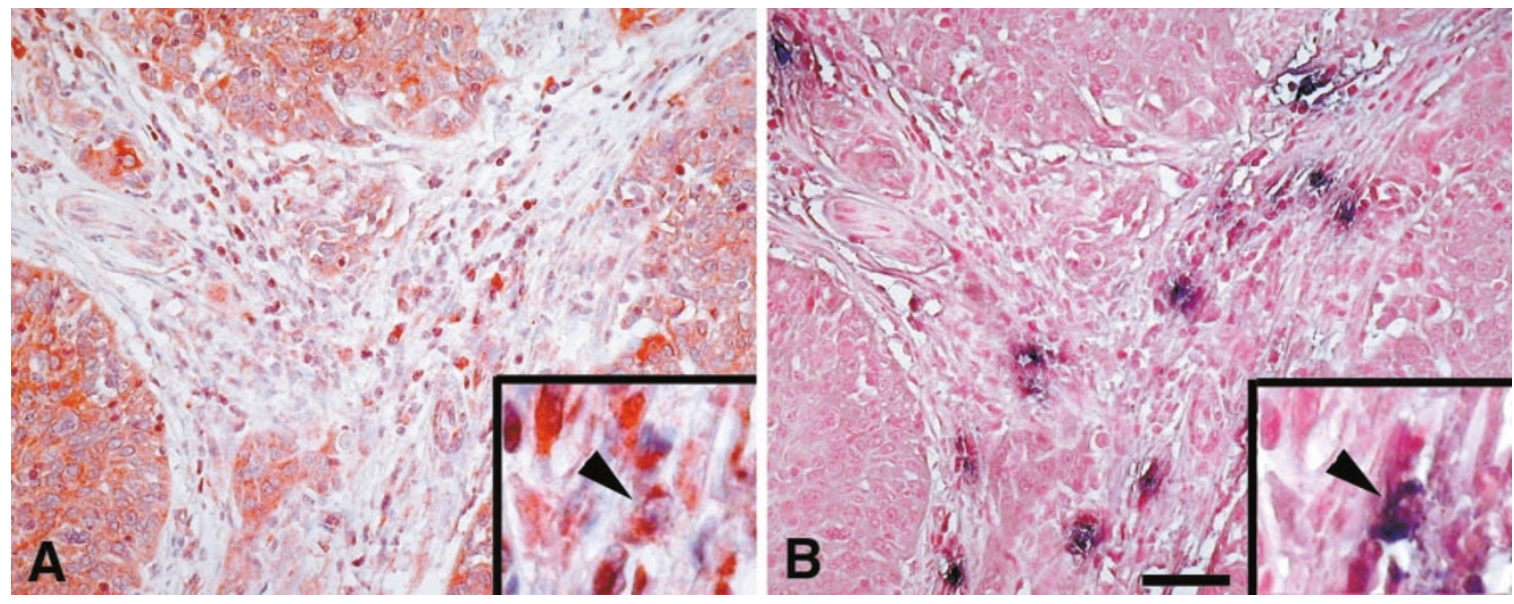

Figure 5 Tryptase staining in mast cells. Double labelling subtraction consecutive immunostaining of the same COX-2 positive tumour section by antiCOX-2 and anti-tryptase antibodies showed that the vast majority (>70\%) of tryptase positive mast cells also express COX-2 Figure 6., insert). Bar: $50 \mu$ m.

gations also by a thorough characterisation of the various cellular subtypes (lymphocytes, endothelial cells, macrophages, etc) in tumour stroma.

From a clinical point of view, it is noteworthy that, while COX2 in tumour cells identifies cervical cancer patients with unfavourable prognosis, COX-2 expression in stroma inflammatory compartment is associated with better clinical outcome, suggesting that COX-2 positive stromal cells can play a role in reducing tumour cell aggressiveness. Therefore, the balance between COX2 expression in the tumour and in the stroma area, rather than the independent evaluation of each compartment, could offer additional prognostic information. In this context, the use of the ratio between COX-2 in the tumour cells and COX-2 in the stroma cells, seems to be a more effective estimate of the cross-talk occurring between tumour tissue and its stromal compartment (Prescott, 2000; Williams et al, 2000). This ratio represents a valuable tool to normalise the amount of COX-2 expression in the tumour on the basis of the status of its stromal component, and to minimize the bias inherent to the use of an arbitrary cut-off.

Indeed, we showed that the ratio between COX-2 in the tumour cells and COX-2 in the stroma cells was very effective in distinguishing patients with low $v s$ high risk of death of disease both in univariate and multivariate analysis. Finally, a very strong corre-

\section{REFERENCES}

Benedetti-Panici P, Maneschi F, Cutillo G, Greggi S, Salerno MG, Amoroso M, Scambia G, Mancuso S (1996) Modified type IV-V radical hysterectomy with systematic pelvic and aortic lymphadenectomy in the treatment of patients with stage III cervical carcinoma. Feasibility, technique and clinical results. Cancer 78: 2359-2365

Bribiesca-Benitez L, Wong A, Utrera D, Castellanos E (2001) The role of mast cell tryptase in neoangiogenesis of premalignant and malignant lesions of the uterine cervix. J Histochem Cytochem 49: 1061-1062

Chao HT, Wang PH, Tseng JY, Lai CR, Chiang SC, Yuan CC (1999) Lymphocyte-infiltrated FIGO Stage IIB squamous cell carcinoma of the cervix is a prominent factor for disease-free survival. Eur J Gynecol Oncol 2: 136-140 Cox DR (1970) Analysis of binary data. London: Methven

Cox DR (1972) Regression models and life tables. J Royal Statist Soc 34: 197 220

Ferrandina G, Lauriola L, Zannoni GF, Fagotti A, Fanfani F, Legge F, Maggiano N, Gessi M, Mancuso S, Ranelletti FO, Scambia G (2002a) Increased cyclooxygenase-2 (COX-2) expression is associated with chemotherapy resistance and outcome in ovarian cancer patient. Ann Oncol 13: $1205-$ 1211 lation between both tumour COX-2 expression and tumour/stroma COX-2 IDV ratio were shown to be highly correlated with response to chemotherapy while, although high COX-2 expression in the stroma was significantly associated with better survival, it failed to directly correlate with response to treatment.

In conclusion, our study showed the usefulness of assessing COX-2 status both in tumour and stroma compartment in order to identify cervical cancer patients endowed with a very poor chance of response to neoadjuvant therapy and unfavourable prognosis. This group of patients could possibly benefit of more individualised treatments, such as selective COX-2 inhibitors, which are already approved for treatment of familial colorectal adenomatous polyposis, and started to be explored in Phase I-II studies (Snyderman, 2001).

\section{ACKNOWLEDGEMENTS}

This work was financially supported by grants from Ministero dell'Università e della Ricerca Scientifica e Tecnologica (MURST) and Italian Association for Cancer Research (AIRC)

Ferrandina G, Lauriola L, Distefano MG, Zannoni GF, Gessi M, Legge F, Maggiano N, Mancuso S, Capelli A, Scambia G, Ranelletti FO (2002b) Increased cyclooxygenase-2 (COX-2) expression is associated with chemotherapy resistance and poor survival in cervical cancer patients. $J$ Clin Oncol 20: 973-981

Gaffney DK, Holden J, Zempolichi K, Murphy KJ, Dicker AP, Dodson M (2001) Elevated cyclooxygenase-2 expression in cervical carcinoma. Reduced cause-specific survival and pelvic control. Am J Clin Oncol 24: $413-416$

Hundley TR, Prasad AR, Beaven MA (2001) Elevated levels of Cyclooxygenase-2 in antigen-stimulated mast cells is associated with minimal activation of p38 mitogen-activated protein kinase. J Immunol 167: 1629-1636

Iniguez MA, Punzon C, Fresno M (1999) Induction of cyclooxygenase-2 on activated $\mathrm{T}$ lymphocytes: regulation of $\mathrm{T}$ cell activation by cyclooxygenase inhibitors. I Immunol 163: $111-119$

Kankkunen J, Harvima I, Naukkariner A (1997) Quantitative analysis of tryptase and chymase containing mast cells in benign and malignant breast lesions. Int J Cancer 72: 385-388 
Kaplan E, Meyer P (1958) Non parametric estimation from incomplete observations. J Am Statist Assoc 53: 457-481

Lehay KM, Ornberg RL, Wang Y, Zwifel BS, Koki AT, Masferrer JL (2002) Cyclooxygenase 2 inhibition by Celecoxib reduces proliferation and induces apoptosis in angiogenic endothelial cells in vivo. Cancer Res 62: $625-631$

Mantel N (1966) Evaluation of survival data and two new rank order statistics arising in its consideration. Cancer Chemother Rep 50: 163-170

Masferrer JL, Leahy KM, Koki AT, Zweifel BS, Settle SL, Woerner BM, Edwards DA, Flickinger AG, Moore RJ, Seibert K (2000) Antiangiogenic and antitumour activities of cyclooxygenase-2 inhibitors. Cancer Res 60: $1306-1311$

Prescott SM (2000) Is cyclooxygenase-2 the alpha and the omega in cancer? Clin Invest 105: $1511-1513$

Ranelletti FO, Almadori G, Rocca B, Ferrandina G, Ciabattoni G, Habib A, Galli J, Maggiano N, Gessi M, Lauriola L (2001) Prognostic significance of cyclooxygenase-2 expression in laryngeal squamous cell carcinoma. Int J Cancer 95: 343-349

Ristimaki A, Sivula A, Lundin J, Lundin M, Salminen T, Haglund C, Joensuu $\mathrm{H}$, Isola J (2002) Prognostic significance of elevated cyclooxygenase-2 expression in breast cancer. Cancer Res 62: 632-635

Santin AD, Ravaggi A, Bellone S, Pecorelli S, Cannon M, Parham GP, Hermonat PC (2001) Tumour-infiltrating lymphocytes contain higher numbers of type 1 cytokine expressors and DR+ T cells compared with lymphocytes from tumour draining lymph nodes and peripheral blood in patients with cancer of the uterine cervix. Gynecol Oncol 81: 424-432

Snyderman M (2001) Biologically useful responses of human neoplasia to Celecoxib and Lovastatin. Proceedings of the American Society of Clinical Oncology (abstract) San Francisco, May 2001
Stolina M, Sharma S, Lin Y, Dohadwala M, Gardner B, Luo J, Zhu L, Kronemberg M, Miller PW, Portanova J, Lee JC, Dubinett SM (2000) Specific inhibition of cyclooxygenase- 2 restores antitumour reactivity by altering the balance of IL-10 and IL-12 synthesis. J Immunol 164: 361 - 370

Tomozawa S, Tsuno NH, Sunami E, Hatano K, Kitayama S, Osada T, Saito S, Tsuruo T, Shibata Y, Nagawa H (2000) Cyclooxygenase-2 overexpression correlates with tumour recurrence, especially haematogenous metastasis of colorectal cancer. Br J Cancer 83: $324-328$

Tsujii M, DuBois RN (1995) Alterations in cellular adhesion and apoptosis in epithelial cells overexpressing prostaglandin endoperoxide synthase 2. Cell 83: $493-501$

Tsujii M, Kawano S, Tsuji S, Sawaoka H, Hori M, DuBois RN (1998) Cyclooxygenase- 2 regulates angiogenesis induced by colon cancer cells. Cell 93: $705-716$

Williams CS, DuBois RN (1996) Prostaglandins endoperoxide synthase: why two isoforms? Am J Physiol 270: $393-400$

Williams CS, Tsujii M, Dey SK, DuBois RN (2000) Host cyclooxygenase-2 modulates carcinoma growth. J Clin Invest 105: 1589- 1594

World Health Organization (1979) WHO handbook for reporting results of cancer treatment. WHO, Geneva 1979 Offset publication no. 48 16-21

Yamada T, Sawatsubashi M, Yakushji H, Itoh Y, Edakuni G, Mori M, Robert L, Miyazaki K (1998) Localization of vascular endothelial growth factor in synovial membrane mast cells: examination with 'multi-labelling subtraction immunostaining'. Virchows Arch 433: $567-570$

Zeid NA, Muller HK (1993) S100 Positive dendritic cells in human lung tumors associated with cell differentiation and enhanced survival. Pathology 25: $338-343$ 